\title{
Embolia pulmonar decorrente de coriocarcinoma metastático com apresentação atípica*
}

\author{
Teresa de Jesus J hayya ${ }^{1}$, Andreia Luisa Simões Francisco ${ }^{1}$, Clystenes Odyr Soares Silva ${ }^{2}$, Rimarcs G. Ferreira ${ }^{3}$
}

É apresentado o caso de uma paciente de 36 anos, com coriocarcinoma metastático pulmonar com apresentação clínica e radiológica atípica. 0 achado de hipertensão pulmonar indicou a possibilidade de tromboembolia pulmonar; todavia, o diagnóstico definitivo e causa da embolia pulmonar foram dados na autópsia. Discutem-se as formas de apresentação das metástases do coriocarcinoma, sua repercussão e o período de latência que pode existir até evidenciar a neoplasia. (J Pneumol 1999;25(6):340-342)

\section{Pulmonary embolism resulting from metastatic choriocarcinoma with atypical presentation: report of a case}

A case is presented of a 36 year-old woman, with metastatic lung choriocarcinoma with atypical clinical and radiological presentation. The finding of pulmonary hypertension indicated the possibility of pulmonary thromboembol ism, still, the definitive diagnosis and cause of pulmonary embol ism were done in the autopsy. The authors discuss the manners of choriocarcinoma metastasis presentation,

its repercussions and the latent period which may exist until it presents evidence of neoplasm.

Descritores - Coriocarcinoma. Embolia pulmonar.

Key words - Choriocarcinoma. Pulmonary embolism.

\section{INTRODUÇÃO}

A doença trofoblástica gestacional é um grupo de entidades nosológicas originadas da proliferação anormal do trofoblasto. 0 coriocarcinoma é a forma de mais alta malignidade, sendo a mola hidatiforme o fator mais importante para o desenvolvimento desta neoplasia ${ }^{(1,2)}$. 0 crescimento primário no útero pode não estar presente ou pode existir sob formas incomuns; por isso, às vezes o diagnóstico é feito tardiamente. Embora, em muitos casos, seja uma doença curável por sua excepcional sensibilidade à quimioterapia(3), a mortalidade ocorre principalmente por falência respiratória, devido a ser mais freqüente a disseminação do coriocarcinoma para o pulmão ${ }^{(4)}$. Raramente, o tumor pode apre-

\footnotetext{
* Trabalho realizado na U niversidade Federal de São Paulo/U nifespEPM.

1. Médica Estágiária da Disciplina de Pneumologia.

2. Professor Adjunto da Disciplina de Pneumologia.

3. Professor Assistente do Departamento de Patologia.

Endereço para correspondência - Clystenes Soares Silva, Universidade Federal de São Paulo/EPM, Disciplina de Pneumologia, Rua Botucatu, 740, 3o andar - 04023-062 - São Paulo, SP. Tel. (011) 576-4238.
}

Recebido para publicação em 9/3/99. Reapresentado em 1/ 6/99. Aprovado, após revisão, em 28/9/99.

sentar-se com embolismo pulmonar e hipertensão pulmonar ${ }^{(5)}$.

0 reconhecimento dessa rara entidade clínica é importante devido ao excelente resultado com tratamento apropriado.

\section{RELATO DO CASO}

Mulher de 36 anos, ex-tabagista, com antecedente de uma gestação com parto normal havia 17 anos; sem história de abortos e em uso de anticoncepcionais orais, sem controle médico, havia 17 anos. Esposo HIV positivo. Q ueixava-se de dispnéia progressiva havia oito meses, concomitante à dor torácica posterior persistente, febre vespertina e tosse não produtiva. 0 caso foi inicialmente tratado como pneumonia e, por ocasião da internação em nosso hospital, a paciente encontrava-se em uso de esquema tríplice, sem haver confirmação bacteriológica de tuberculose. Ao exame físico apresentava-se com palidez cutâneo-mucosa, cianose periférica que melhorava com repouso, afebril, FC de $72 \mathrm{bpm}$, FR de $42 \mathrm{rpm}$, ingurgitamento jugular, bulhas normorrítmicas e normofonéticas com sopro sistólico em foco mitral, irradiado para foco tricúspide. $\mathrm{Na}$ ausculta pulmonar evidenciavam-se murmúrio vesicular diminuído, estertores inspiratórios na base esquerda e sibilos difusos. Gasometria arterial em ar ambiente: hipoxemia e alcalose respiratória. $\mathrm{Hb}=10,7 \mathrm{~g}, \mathrm{Ht}=35 \%$, plaquetas $=329.000 / \mathrm{mm}^{3}$, leucócitos $=31.800 / \mathrm{mm}^{3}(B=$ $5 \%, \mathrm{~S}=80 \%, \mathrm{~L}=8 \%$ ), VHS $=105 \mathrm{~mm} / \mathrm{h}, \mathrm{AP}=89 \%$, química sanguínea normal. Radiograma (Figura 1) e tomografia 


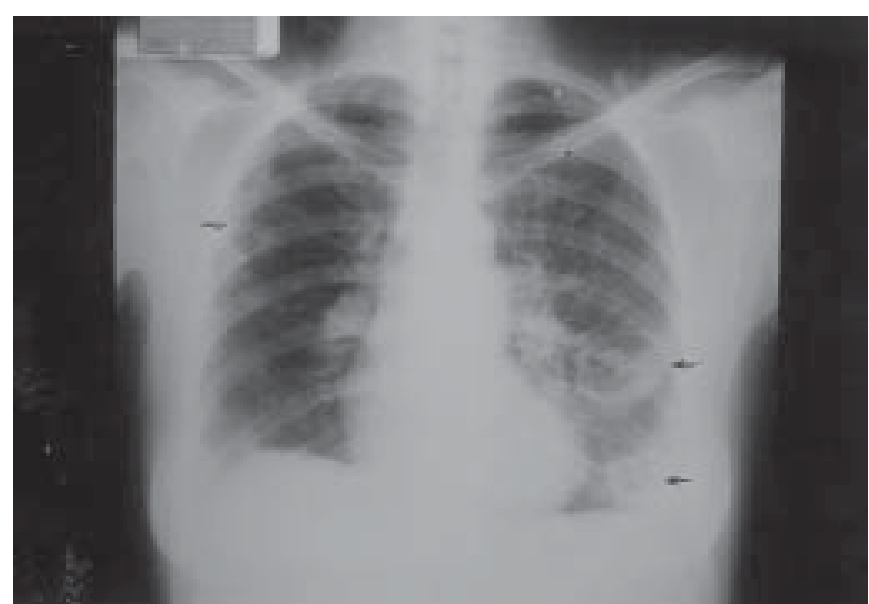

Figura 1 - Radiograma de tórax apresentando duas cavidades em lobo inferior de pulmão esquerdo e uma em lobo superior direito

computadorizada de tórax: duas cavidades no lobo inferior esquerdo e uma no lobo superior de pulmão direito. Pesquisa de BAAR em escarro, provas imunológicas e HIV, negativos. Biópsia transbrônquica: "necrose de coagulação, colapso alveolar e fibrose focal", BAAR, fungos e P. carinii, negativos. ECG normal. Ecocardiograma: discreto aumento das cavidades direitas, insuficiência mitral moderada, pressão de artéria pulmonar de $65 \mathrm{mmH} \mathrm{g}$, o que sugeriu tromboembolismo pulmonar. Durante esse período a paciente foi deteriorando-se, sendo necessária admissão na UTI e ventilação mecânica não invasiva. Realizada biópsia por minitoracotomia, achando-se pulmão friável, amolecido e com múltiplas cavidades, evoluindo para óbito no pós-operatório. Achados da autópsia: distúrbios da coagulação (fenômenos tromboembólicos), trombo neoplásico ocluindo o lume de ramos da artéria pulmonar, vasculites em ramos pulmonares e renais, infartos cavitários bilaterais, edema pulmonar, hemorragia pulmonar, cor pulmonale crônico, congestão visceral generalizada (Figuras 2 e 3). Diagnóstico: coriocarcinoma metastático pulmonar; causa imediata de óbito: embolia pulmonar neoplásica maciça.

\section{DISCUSSÃO}

O coriocarcinoma faz parte de um grupo de doenças trofoblásticas. A metade dos casos é precedida por mola hidatiforme, cujo diagnóstico precoce e tratamento adequado possibilitam cura. É uma neoplasia maligna caracterizada por um padrão dimórfico dos elementos trofoblásticos na ausência de vilosidades coriônicas ${ }^{(1-3)}$. A maior prevalência ocorre em mulheres entre 20 e 40 anos ${ }^{(4,6)}$. A relação do coriocarcinoma com qualquer tipo de gestação tem sugerido malignização das células sinciciotrofoblásticas (molar ou não) após metastatizar ${ }^{(2,7)}$, porém, aparentemente, existe um período de latência entre a gestação causal e 0 aparecimento da neo-

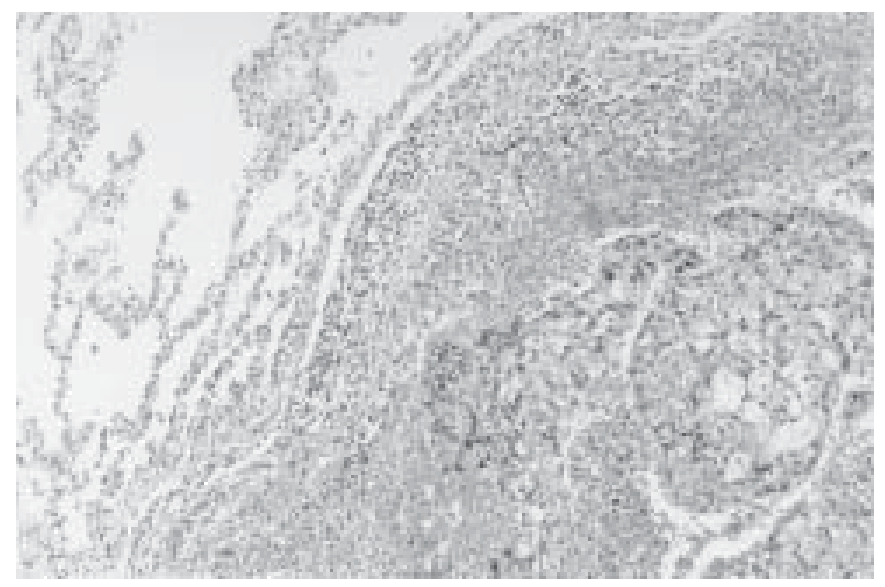

Figura 2 - Pulmão apresentando oclusão vascular por material hipercelular, permeado por hemácias (HE, 100x)

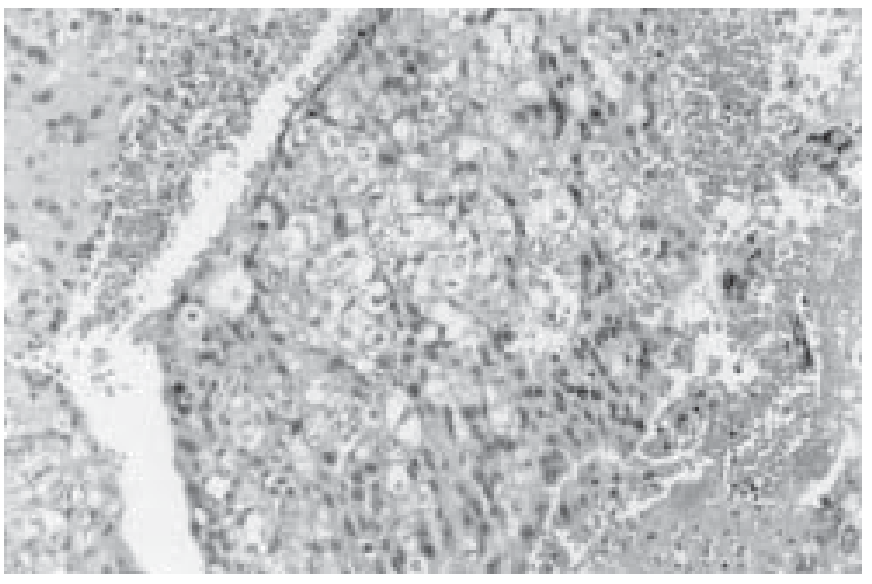

Figura 3 - O clusão vascular por dupla população celular, composta por células com citoplasma amplo e claro e por células com citoplasma eosinofílico, ambas pleomórficas, com núcleos irregulares, representando, respectivamente, cito e sinciciotrofoblasto (HE, 400x)

plasia, que pode ser de semanas até vários anos $(>10 \text { anos })^{(4,8)}$ e inclusive apresentando-se na pós-menopausa( ${ }^{(9)}$, o que torna difícil a suspeita diagnóstica sem antecedentes recentes; têm sido descritos casos de coriocarcinoma "não gestacional", muito raro, originado de células germinativas gonadais e extragonadais $s^{(1,8)}$. Às vezes, o sítio primário do coriocarcinoma pode não existir, compreendendo-se uma involução espontânea após metastatizar ${ }^{(2,10)}$, descrito em 10 a 50\% dos casos $^{(6)}$; em menos de $1 \%$ dos casos há remissão espontânea das metástases(2). No presente caso houve remissão espontânea do primário e a metástase pulmonar foi evidenciada pela presença de trombo neoplásico ocluindo o ramo da artéria pulmonar. Existe a possibilidade de haver tido aborto espontâneo que haja passado despercebido ou poderia tratar-se de um período de latência prolongado.

Há alta incidência de metástases para vários órgãos, sendo o pulmão acometido em mais de $90 \%$ dos $\operatorname{casos}^{(4,11)}$. São 
descritas três formas básicas de metástases pulmonares: 1) típicas, mais comuns e às vezes associadas a cavitações, 2) alveolares; e 3) embólicas e infartos pulmonares secundários a obstrução arterial, que têm sido detectadas em $20 \%$ dos

\section{REFERÊNCIAS}

1. Tomoda Y, Ishizuka T, Goto S, Furuhashi Y. Trophoblastic disease. Curr Top Pathol 1992;85:203-230.

2. Baergen R. Gestational choriocarcinoma. Gen Diagn Pathol 1997; 143:127-142.

3. Lurain J. Management of high-risk gestational trophoblastic diseases. J Reprod Med 1998;43:44-52.

4. A wotedu A, Odunfa A, Aghadiuno P, Ogunlesi A, et al. Pulmonary metastatic malignant tumors in Ibadan, Nigeria: 10 years autopsy review. Cent Afr J Med 1991;37:285-289.

5. Libshitz $\mathrm{H}$, Baber $\mathrm{C}, \mathrm{Hammond} \mathrm{Ch}$. The pulmonary metastases of choriocarcinoma. Obstet Gynecol 1977;49:412-415.

6. Cruz H, Lopez J , Alcantara A, J astrow L, Miranda H. Coriocarcinoma gestacional avanzado. Estudio clínico patológico de 40 casos. Ginecol Obstet Méx 1994;63:384-388.

7. Ober W, Edgcomb J, Price E. The pathology of choriocarcinoma. Ann N Y Acad Sci 1971;172:299-426.

8. De Morais C, de Carvalho C, Barbas Filho J. Coriocarcinoma pulmonar metastático (endarterial). Rev Hosp Clín Fac Med Univ São Paulo 1983;38:218-221. casos e que podem evoluir para cavitação(5,12). Os pacientes podem desenvolver hipertensão pulmonar reversível ou cor pulmonale agudo ou progressivo, dependendo do calibre e do tipo de comprometimento dos vasos pulmonares ${ }^{(13,14)}$.

9. Dougherety C, Cunningham C, Mickal A. Choriocarcinoma with metastasis in a postmenopausal woman. Am J Obstet Gynecol 1978; 132:700-701.

10. Bardawill W, Toy B. The natural history of choriocarcinoma: problems of immunity and spontaneous regression. Ann N Y Acad Sci 1959; 80:197-199.

11. Soares F. Aspectos clínicos e morfológicos da embolia pulmonar neoplásica. J Pneumol 1991;17:127-140.

12. Libshitz H, North L. Pulmonary metastases. Radiol Clin North Am 1982;20:437-451.

13. Yutani Ch, Imakita M, Ishibashi-U eda $H$, Katsuragi M, Yoshioka $T$, Kunieda T. Pulmonary hypertension due to tumor emboli: a report of three autopsy cases with morphological correlations to radiological findings. Acta Pathol J pn 1993;43:135-141.

14. Seckl M, Rustin G, Newlands E, Gwyther S, Bomanji J. Pulmonary embolism, pulmonary hypertension, and choriocarcinoma. Lancet 1991;338:1313-1315. 